\title{
AN ELECTRICAL METHOD OF MEASURING SMALL INTERVALS OF TIME.
}

\author{
By F. C. BRown.
}

$\mathrm{W}^{\mathrm{H}}$

HEN it is desired to measure the time between two mechanical operations, we have found that it may be carried out quite accurately by the use of resistances, battery, ballistic galvanometer, condenser, and contact keys. The principle is based on the relation between the electro-magnetic and the electro-static systems of units, and if we define the ohm in terms of the dimensions of a mercury column, the method is an absolute one. Aside from the advantage of having an absolute measure of time in terms of length, or of length, mass and resistance, the advantages of the method are simplicity and ease of manipulation. The interval of time to be measured may be diminished to the limits of dependable electrical contact, no doubt less than o.oor second. How large the interval may be varies from 0.4 second to several seconds depending on the period of the galvanometer.

\section{THEORY.}

The theory of the method is essentially the same as that given in a previous paper on a method of measuring fluctuating resistances, ${ }^{1}$ but as Dr. Erich Schneckenburg, of Berlin, has kindly pointed out that the method of derivation of equation (I) in that paper is not obvious, I shall briefly review the essential steps.

Suppose it is desired to know the interval of time, $\Delta t$, between the making of electrical contact at $t_{1}$, and the time of breaking of contact at $t_{2}$. It is to be shown that this time interval varies directly as the throw of a ballistic galvanometer which has been connected, as shown in Fig. I, in a Wheatstone's bridge circuit during the interval.

Considering the battery resistance small compared to the other resistances, we obtain, by Kirkchhoff's and Ohm's Laws, the current through the galvanometer to be

where

$$
i=\frac{E(a R-b x)}{\left(k_{1} x+k_{2}\right)^{2}},
$$

$$
\begin{aligned}
& k_{1}=G b+a G+a b+a R+b R, \\
& k_{2}=a R G+b R G+a b R .
\end{aligned}
$$

${ }^{1}$ By Brown and Clark, Phys. Rev., XXXIII., p. 53, I9II. 
By differentiating equation (I) with regard to $x$, we have

$$
\frac{d i}{d x}=-\frac{E\left(b k_{2}+k_{1} a R\right)}{\left(k_{1} x+k_{2}\right)^{2}} .
$$

We may write this equation in the following form and keep within the accuracy of laboratory experimentation,

$$
\Delta i=-\frac{E\left(b k_{2}+k_{1} a R\right) \cdot \Delta x}{\left(k_{1} x+k_{2}\right)^{2}},
$$

where it is understood that if the value of $\Delta x$ is relatively too large a small correction must be added.

Now if it is agreed that $\Delta x$ is to be measured as the amount of variation of $x$ from that value which gives no current through the galvanometer as required by equation ( $\mathrm{I}$ ), then obviously $\Delta i=i$, and since the negative sign is of no importance,

$$
i=\frac{E\left(b k_{2}+k_{1} a R\right) \cdot \Delta x}{\left(k_{1} x+k_{2}\right)^{2}} .
$$

Since the quantity of electricity that passes through the galvanometer as the result of the closed circuit during the interval $\Delta t$ is $Q=i \cdot \Delta t$, and since in a ballistic galvanometer $K \cdot d=i \cdot \Delta t$, where $K$ is the usual constant as obtained by a standard condenser and a known electromotive force, it follows that,

$$
\Delta t=\frac{K \cdot d \cdot\left(k_{1} x+k_{2}\right)^{2}}{\Delta x \cdot E \cdot\left(b k_{2}+k_{1} a R\right)} .
$$

The only qualification, that should be made to this result is, that either the galvanometer coil must not have moved over an appreciable distance during the time interval to be measured, or that the effective resistances in the closed circuit should be so large that the damping action can be neglected. I take it that it is quite generally understood that self induction in the coil of a ballistic galvanometer can be disregarded. At any rate the experimental results in this paper will obviously warrant the assumption.

If a standard condenser of capacity $C$ is charged by the same electromotive-force $E$, and then discharged through the same galvanometer, thereby giving a deflection $d^{\prime}$, we may write equation (5) in the form,

$$
\Delta t=\frac{C \cdot d \cdot\left(k_{1} x+k_{2}\right)^{2}}{\Delta x \cdot d^{\prime} \cdot\left(b k_{2}+k_{1} a R\right)} .
$$

We therefore have the interval of time between the two contacts 
measured in terms of the units of length and the units of resistance, and if we choose to define the unit of resistance in terms of the dimensions and the mass of a mercury column, our fundamental system of units may be regarded as solely based on the units of length and of mass. The relative merits of the unit of time as a derived unit will be discussed later.

\section{Experimental Test.}

In order that the method may be workable it is sufficient to show that the time interval, between contacts at $t_{1}$ and at $t_{2}$, varies directly as the deflection of the galvanometer, and that the computed value of the interval agrees with that observed. In our former paper the relation between the deflection and the time was recorded, but the linear relation did not hold for greater values of the time than 0.2 second. The lack of agreement beyond this point has since been found to have arisen from inaccurate theoretical calibration of the timing pendulum, and not to the fact that the galvanometer coil was not in its zero position while the entire quantity of electricity was impressed, as was previously supposed. Using correct values of the time as obtained by an experimental calibration of the pendulum, Messrs. T. Ingwaldson and J. S. Hilliard of our laboratory have obtained the observations shown on the curve in Fig. 2. Each point shown on the curve is the average of four observations. The accompanying table gives a fair representation of the reliability of the observations for a single setting of the keys. However it was found at the close of the observations that there was an error of about one $\mathrm{mm}$. in the throw of the galvanometer due to the inaccurate setting of the keys.

TABLE I.

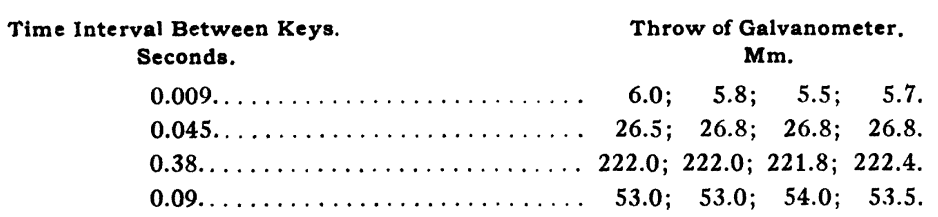

The indicated values of the resistances used for balancing the bridge were, $a=b=x=9,000 \mathrm{ohms}$, and $R=9,004.6 \mathrm{ohms}$. The source of electromotive force was a storage battery of 38.0 volts. The value of $R$ indicates the probable error in the resistances. In order to obtain the observations given above the value of $x$ was changed to 9, Ioo, thereby giving a value of $\Delta x=100 \mathrm{ohms}$. The resistance of the galvanometer was $950 \mathrm{ohms}$, and its period was 26.7 seconds.

The linear relation between the throw of the galvanometer and the time holds between 0.002 second and 0.32 second as accurately as the 
particular apparatus was capable of testing. Of course for longer periods of time there would appear appreciable errors due to the swinging of the galvanometer coil in a closed circuit of high resistance. It is possible to correct for this error or to diminish it greatly by increasing the resistances and by increasing the period of the galvanometer. By increasing the mass of the galvanometer coil without increasing its inductance, $i$. e., such as is done by adding weights to the coil, will clearly doubly lessen the relative error due to the cause above mentioned. But as adding mass in this way also decreases the sensibility, I am not prepared to say how far the last idea could be carried satisfactorily. To show the adaptability of the method for measuring intervals of time larger than those given in Table I., the period of the galvanometer coil was increased to $9 \mathrm{I}$ sec. by using a phosphor bronze strip suspension I meter in length.

TABLE II.

Deflections.

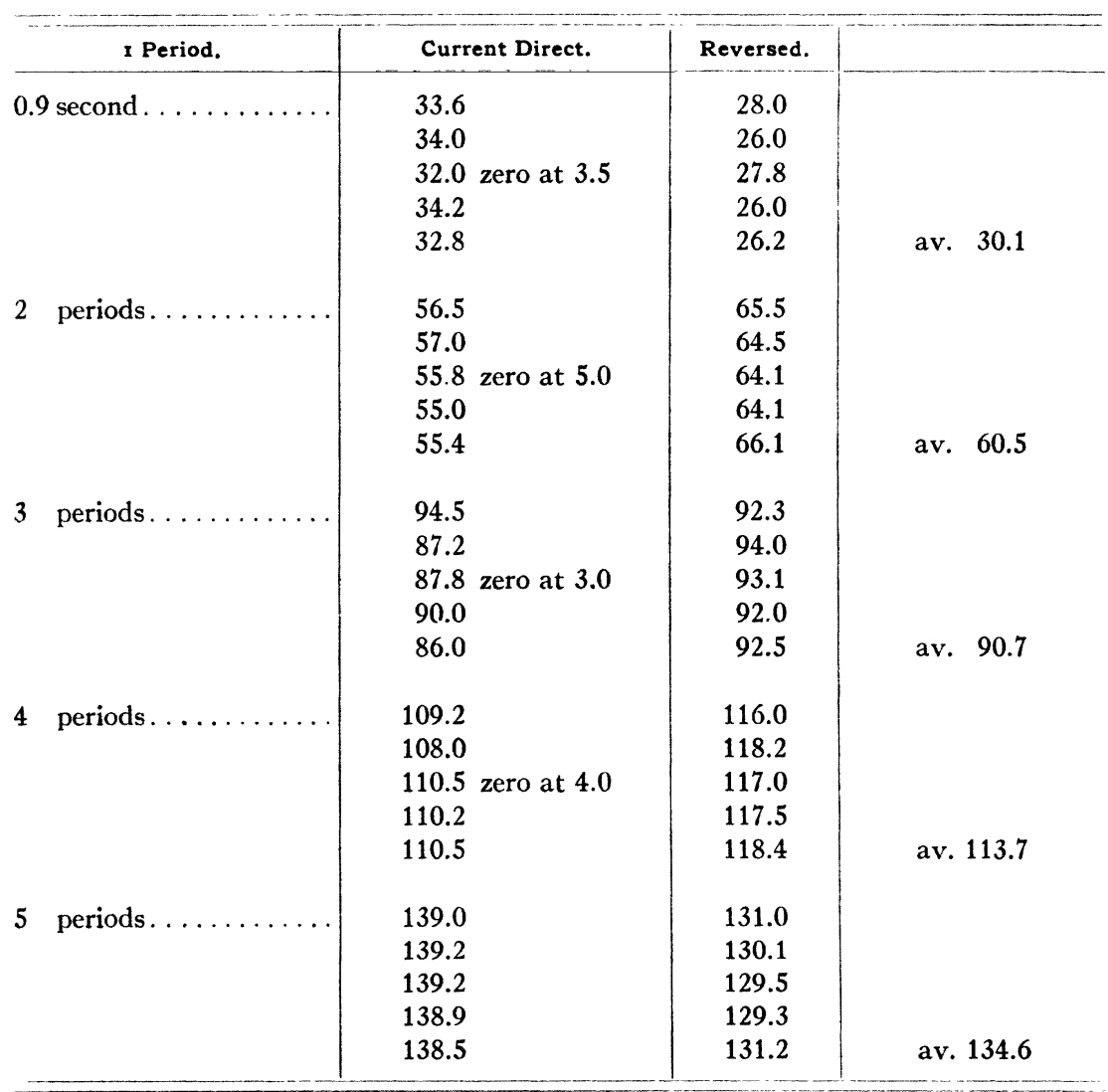


The time interval was measured in terms of the number of swings of the pendulum. The keys were knocked down always at the middle of the arc. The period was about 0.9 second and the intervals were therefore some multiple of $0.9 \mathrm{sec}$. The observations are shown in Table II. The resistances used for balance in the bridge were 9,000 ohms each, and $\Delta x$ was 500 ohms. A single dry cell was in circuit. When the galvanometer period is so large it is highly advisable to determine the zero position from the amplitude readings to the right and left such as is practiced in balance work. After the zero position is found it is very easy to bring the coil to rest.

It is observed that up to 3 periods,

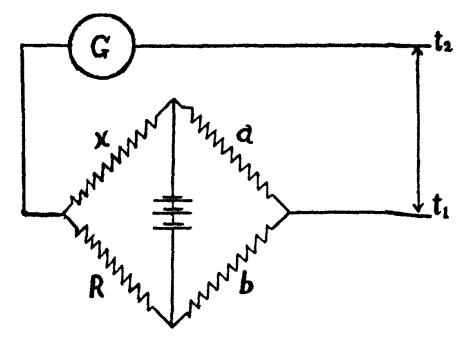

Fig. 1. i. e., 2.7 seconds, the deflection is proportional to the time interval, but beyond this the deflection falls off quite rapidly.

However by replacing the $9,000 \mathrm{ohm}$ resistances in $a$ and $b$ (see Fig. I) with I00,000 $\mathrm{ohm}$ values each, the deflection was proportional to the time up to about 3.6 seconds as shown in the following table, where each recorded value is the average of ten observations.

TABLE III.

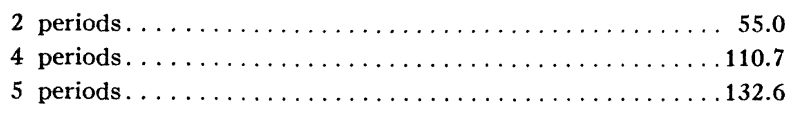

The foregoing results would seem to indicate that for large intervals of time it is more important to have a long period than it is to have extremely high resistances.

In order to test the adaptability of the method for absolute determinations of time, the constant of the galvanometer as obtained by equation (5) was compared with the value as obtained by the well-known battery and condenser method. By the current method the value was calculated to be $K=1.77 \times \mathrm{IO}^{-10}$ coulombs per $\mathrm{mm}$. and by the second method, $K=1.82 \times 10^{-10}$. This variation is quite within the limits of error of the capacity and the resistances used. As we did not have a standard condenser or resistances of the highest accuracy, it was not considered worth while to investigate the possible accuracy of the method any further.

As a practical laboratory method of measuring small intervals of time I believe that the scheme presented should be useful in certain kinds of 
work, wherever it is feasible to mark time by electrical contacts. We believe that it is advisable to reserve in every laboratory a ballistic galvanometer of very slow period primarily for measuring time intervals. By posting at this galvanometer particular values of resistance and of

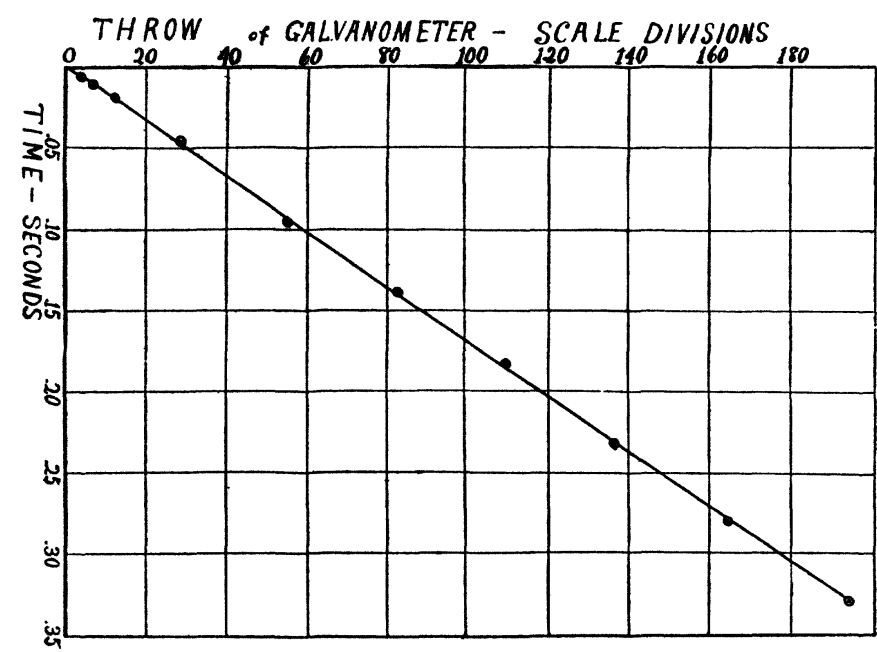

Fig. 2.

electromotive force to be used, and also the equivalent time interval, as calculated, for one division deflection, the troubles of calculations can be eliminated once for all. By taking only the most elementary precautions therefore the method should in many cases supplant a chronograph and standard clock. From this point of view the simplicity and quickness of manipulation are striking.

If it is desired to measure two or more time intervals simultaneously, several galvanometers and sets of keys may be connected in the same bridge circuit. The use of two galvanometers is illustrated, as used for measuring fluctuating resistances, in my previous paper on the recovery of the Giltay selenium cell and the nature of light-action in selenium. ${ }^{1}$ If one observer wishes to read several time intervals simultaneously, he can do so by altering the periods of the galvanometers sufficiently so that he can have time to record each deflection.

\section{The Advisability of the Second as a Derived Unit.}

Quite apart from the usefulness in the laboratory, are the considerations concerning the practicability of the method for determining the absolute unit of time. In this discussion any of the well-known methods used

1 Phys. Rev., XXXIII., p. 412, 1911. 
by standard laboratories might be substituted. The only question is whether it is best to have the second as a fundamental or a derived unit of time. A fundamental absolute unit should have two distinct merits. It should be one capable of being reproduced to a sufficiently high degree of accuracy, and it should be unvarying for all time. As is well known our present fundamental absolute unit of time fulfills the first requirement remarkably well, but there is considerable doubt as to how long it will remain fixed. Tidal action, the shrinkage of the earth, friction with meteorites and friction of the atmosphere with itself, in addition to friction of possible electromagnetic origin, all tend not only to vary the value of the second, but also to make it almost a hopeless task to satisfactorily correct the unit for future periods of time.

By way of comparison, there is no question but that we cannot reproduce the unit of resistance, and consequently the derived unit of time, with as great accuracy as we can reproduce the present absolute fundamental unit of time, but on the other hand we have no information that would lead us to believe that the unit of resistance, defined in terms of the dimensions and mass of a mercury column, would not remain fixed.

It seems wise to suggest therefore that it would be worth considering the advisability of adopting our present unit as a secondary standard, which might be rechecked and revised in the distant future, if the electrical method, with the ohm as the fundamental unit, should show the value of the second to be materially in error. At any rate the temporary establishment of the unit of time defined from as many sources as possible might lead to discoveries of far-reaching importance.

The Physical laboratory,

The State University of Iowa. 